\title{
Synthesis, Structure, and Mechanical Properties of A-B-A Tri-Block Copolymers Consisting of Poly $(\varepsilon-N$-benzyloxy- carbonyl-L-lysine) as the A Component and Polybutadiene as the B Component
}

\author{
Kouhei KugO, Toshio HAYASHI, and Akio NAKAJIMA \\ Department of Polymer Chemistry, Kyoto University, \\ Sakyo-ku, Kyoto 606, Japan.
}

(Received December 28, 1981)

\begin{abstract}
A-B-A type tri-block copolymers composed of $\varepsilon$ - $N$-benzyloxycarbonyl-L-lysine as the $\mathrm{A}$ component and butadiene as the B component were prepared. The synthesis could be carried out by allowing the $N$-carboxy anhydride of the $\alpha$-amino acid to react with the amineterminated polybutadiene. The structure of these block copolymers was estimated using the relation between the helix-coil transition temperature and the number-average molecular weight. The morphology of the membrane specimens was investigated by wide-angle X-ray diffraction and electron microscopy. It was found that the dynamic mechanical spectra can be well explained by the microheterophase structure observed under electron microscope. By minimizing the Gibbs free energy $\Delta G$ per unit volume for the micelle formation, the domain size was estimated for each type of micelle. The shape and size calculated were in accord with those observed by electron microscope.

KEY WORDS Tri-Block Copolymer / Poly $(\varepsilon-N$-benzyloxycarbonyl-Llysine) / Polybutadiene / Microheterophase Structure / Dynamic Mechanical Properties /
\end{abstract}

Recently, Gallot et al. ${ }^{1,2}$ carried out a study on the synthesis and structure determination of A-B type di-block copolymers consisting of polypeptides and polymers such as vinyl polymers and polybutadiene.

In previous studies, ${ }^{3-5}$ we synthesized A-B-A type tri-block copolymers consisting of $\gamma$-benzyl Lglutamate as the A component and butadiene as the B component, and characterized the bulk properties of their membranes: microheterophase structure, mechanical properties, and water permeability, etc. The results of circular dichroism measurements in ethylene dichloride solution and infrared spectra measurements in the solid state showed that the polypeptide chain in the copolymer assumes the same $\alpha$-helical conformation as the homopolypeptide chain. The microheterophase structure in membranes cast from different solvents was examined by electron microscope. The micelle dimensions evaluated from thermodynamic considerations, taking into account the conformational parameters of the component block chains, were in good agreement with those obtained from electron micrographs. The dynamic mechanical spectra for the block copolymer membranes predicted accurately the morphology of spherical and cylindrical micelles when analyzed by Takayanagi's equivalent mechanical model. ${ }^{6}$ Moreover, from the experimental results on the hydraulic permeability of water, it was clarified that the interfacial region between the $\alpha$-helical polypeptide chain and the amine-terminated polybutadiene chain is responsible for the water permeability.

In order to confirm whether these findings are general characteristics of block copolymers consisting of a polypeptide block and a polybutadiene block, we studied the preparation, structure, and properties of A-B-A type tri-block copolymers (LBL) composed of poly $(\varepsilon-N$-benzyloxycarbonyl-Llysine) (PBCL) as the A component and polybutadiene (PB) as the $\mathrm{B}$ component. The results are described in this paper. The surface properties of 
these block copolymer membranes will be reported in the following paper.

\section{EXPERIMENTAL}

Synthesis and Purification of Materials AmineTerminated Polybutadiene

The preparation and purification of the middle block, a cycloaliphatic secondary amine-terminated polybutadiene (ATPB), are described in a previous paper. ${ }^{3}$ The ATPB was high in trans isomers, having a number-average molecular weight, $M_{n}$, of 3600 .

\section{$\varepsilon$ - $N$-Benzyloxycarbonyl-L-lysine $N$-Carboxy Anhy-} dride

The mononer $N$-carboxy anhydride of $\varepsilon-N$ benzyloxycarbonyl-L-lysine ( $\varepsilon$-BCL-NCA) was prepared according to the method proposed by Blout and Karlson, ${ }^{7}$ and purified by recrystallization from an ethyl acetate solution with the addition of petroleum ether. Recrystallization was repeated from two to three times.

\section{Synthesis of Block Copolymers}

Calculation was made of the amount of $\varepsilon$-BCLNCA and ATPB necessary for obtaining the desired degree of polymerization of the polypeptide block. The polymerization was carried out in the absence of moisture, at room temperature, in a dioxanemethylene dichloride mixture $(1: 2, \mathrm{v} / \mathrm{v})$ at a total concentration of $\varepsilon$-BCL-NCA and ATPB, $3 \%$. After $48 \mathrm{~h}$, the polymerization was terminated, and the copolymer was precipitated by 5 volumes of cold methanol. This method of precipitation eliminated any traces of $\varepsilon$-BCL-NCA still present and the oligopeptide formed by autopolymerization. ${ }^{8}$ The copolymer was then dried in vacuo at $50^{\circ} \mathrm{C}$.
The results of the copolymerization are listed in Table I.

\section{Characterization. Molecular Weights and Molecular Weight Distributions}

The molecular weights of the tri-block copolymers were estimated from elemental analysis using the number-average molecular weight, $M_{n}=3600$, of ATPB. The molecular weights obtained are, therefore, expected to be the number-average molecular weights. The number-average molecular weight of PBCL was determined from the limiting viscosity number $[\eta]$ in $m$-cresol measured with a Ubbelohde type viscometer; the $[\eta]$-number-average molecular weight $M_{n}$ relationship ${ }^{9}$ proposed for PBCL was used. The limiting viscosity numbers $[\eta]$ of the block copolymers were measured using the same viscometer under the same conditions. The limiting viscosity numbers $[\eta]$ and molecular weights obtained are summarized in Table I. The molecular weight evaluated from elemental analysis was checked in $N, N$-dimethylformamide at $25^{\circ} \mathrm{C}$ by using a MOM Type-3170-b ultracentrifuge. Molecular weight distributions of these polymers were examined by sedimentation patterns.

\section{Composition of Block Copolymers}

The molar composition of the LBL block copolymers was determined by elemental analysis and the number-average molecular weight of ATPB. The results are listed in Table I.

Conformation and Helix-Coil Transition of the Polypeptide Blocks

Circular dichroism (CD) spectra were measured at $25 \pm 0.5^{\circ} \mathrm{C}$ on a JASCO J-20 CD/ORD spectropolarimeter equipped with a quartz cell having a

Table I. Molecular characterization of samples prepared

\begin{tabular}{|c|c|c|c|c|c|}
\hline \multirow{2}{*}{ Designation } & \multirow[b]{2}{*}{$\begin{array}{c}\mathrm{dl} \mathrm{g}^{-1} \\
\left(m \text {-cresol, } 25^{\circ} \mathrm{C}\right)\end{array}$} & \multirow{2}{*}{$\frac{\text { Butadiene }}{\mathrm{mol} \%}$} & \multirow{2}{*}[\theta]{$_{222}^{c} /[\theta]_{222}^{0}$} & \multirow{2}{*}{$P_{\mathrm{A}}$} & \multirow{2}{*}{$M_{n} \times 10^{-}$} \\
\hline & & & & & \\
\hline LBL-1 & 0.33 & 52.3 & 0.47 & 28 & 1.8 \\
\hline LBL-2 & 0.39 & 42.3 & 0.57 & 42 & 2.6 \\
\hline LBL-3 & 0.47 & 23.7 & 0.76 & 98 & 5.5 \\
\hline LBL-4 & 0.48 & 16.2 & 0.81 & 158 & 8.7 \\
\hline LBL-5 & 0.49 & 11.9 & 0.88 & 226 & 12.2 \\
\hline PBCL & 1.83 & 0.0 & 1.00 & 795 & 41.7 \\
\hline
\end{tabular}


path length of $1 \mathrm{~mm}$. The optical rotatory dispersion (ORD) spectra were measured to examine the helix-coil transition, using a Yanagimoto OR-100 Type spectropolarimeter.

\section{Wide-Angle X-Ray Diffraction and Electron Microscopy \\ Wide-angle X-ray diffraction (WAXD) profiles} were obtained by using $\mathrm{Ni}$-filtered $\mathrm{Cu} K \alpha$ radiation, setting a flat surface of the membrane normal to the beam. Films for WAXD measurements were cast from a 10:1 (v/v) mixture of chloroform (CF) and 2,2,2-trifluoroethanol (TFE). In observing the microheterophase structure with a Hitachi H-500 transmission electron microscope, thin membranes cast from the same solvent mixture as that for WAXD measurements were stained with osmium tetroxide vapor.

\section{Mechanical Property}

For mechanical studies, films of $c a .50 \mu \mathrm{m}$ in thickness were prepared by casting from the same solvent as that for the $\mathrm{X}$-ray diffraction experiments. The dynamic mechanical relaxation behavior was measured with a DDV-II Rheovibron at a frequency of $110 \mathrm{~Hz}$ and a heating rate of $0.3^{\circ} \mathrm{C}$ $\min ^{-1}$ over a temperature range from -20 to $100^{\circ} \mathrm{C}$.

\section{RESULTS AND DISCUSSION}

\section{Molecular Characterization}

The pertinent data on polymerization are summarized in Table I. From the circular dichroism (CD) spectra in 1,2-dichloroethane (DCE), the ratios of the residue ellipticity $[\theta]_{222}$ of LBL block copolymers to that of PBCL homopolymer, $[\theta]_{222}^{c} /$ $[\theta]_{222}^{0}$, were evaluated and are listed in the third column of Table I. The value of the ratio is in accord with that of molar composition obtained from elemental analysis. This fact shows that the helical content of the $\mathrm{L}$ component in the LBL block copolymers is equal to that of the PBCL homopolymer in DCE solution. In Table I, $P_{\mathrm{A}}$ is the degree of polymerization of the A block chain.

The sedimentation patterns of LBL-5 and LBL-3 block copolymers and the PBCL homopolymer exhibit a single peak and no tailing on either side of the peak, as shown in Figure 1. This behavior was observed for all block copolymers studied.
(1)

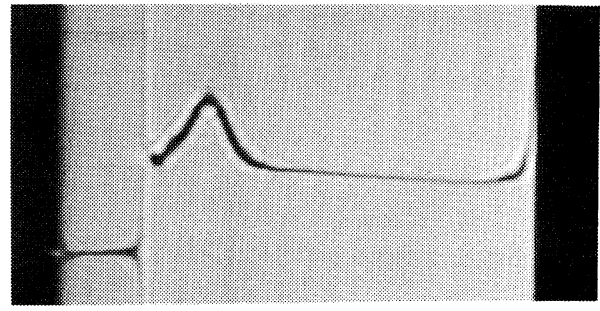

(2)

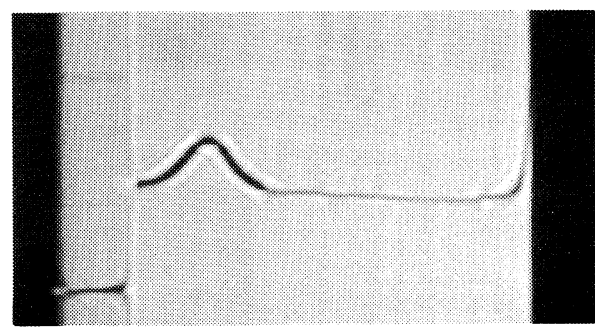

(3)

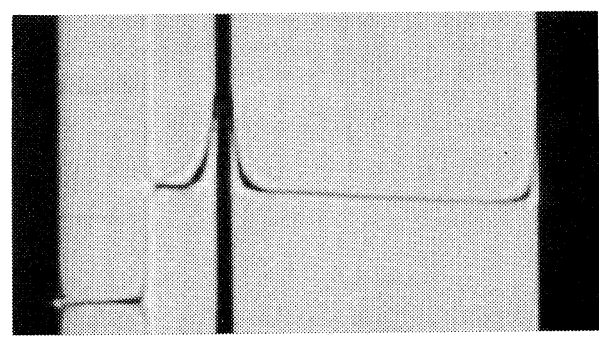

Figure 1. Sedimentation patterns of samples in DMF at $25^{\circ} \mathrm{C}$ under the following conditions: speed of rotation, 50,000 rpm, Schlieren angle, $65^{\circ}$, and concentration, $0.5 \mathrm{~g} \mathrm{dl}^{-1}$; (1) LBL-5 (70 min); (2) LBL-3 (85 min); (3) PBCL (80 min).

The number-average molecular weight $M_{n}$ of LBL-5 was examined by ultracentrifugation for comparison with the $M_{n}$ evaluated from elemental analysis. For a polydisperse system, the apparent weight-average molecular weight $M_{w}^{\text {app }}$ and the apparent $z$-average molecular weight $M_{z}^{\text {app }}$ are determined as functions of the polymer concentration. We assumed that the small amount of the polybutadiene portion would not effect the thermodynamic nonideality. From the LBL-5 measurements, we obtained $M_{w}^{\mathrm{app}}=14.4 \times 10^{4}$ at $C_{0}=0.06 \mathrm{~g} \mathrm{dl}^{-1}$, $13.8 \times 10^{4}$ at $C_{0}=0.14 \mathrm{~g} \mathrm{dl}^{-1}, 14.1 \times 10^{4}$ at $C_{0}=0.15$ $\mathrm{g} \mathrm{dl}^{-1}$, and $13.9 \times 10^{4}$ at $C_{0}=0.22 \mathrm{~g} \mathrm{dl}^{-1}$, in which $C_{0}$ is the polymer concentration before centrifugation. By extrapolation to infinite dilution, we obtain the weight-average molecular weight, $M_{w}=$ $14.5 \times 10^{4}$. Furthermore, the apparent $z$-average molecular weight $M_{z}^{\text {app }}$ at $C_{0}=0.22 \mathrm{~g} \mathrm{dl}^{-1}$ was 
evaluated, and then we obtained $M_{z}^{\mathrm{app}} / M_{w}^{\mathrm{app}}=1.15$. We assumed that $M_{z}^{\text {app }} / M_{w}^{\text {app }}$ is nearly equal to $M_{z} /$ $M_{w}$, and obtained $M_{n}=12.3 \times 10^{4}$ for the numberaverage molecular weight of LBL-5 from these data, assuming the generalized exponential distribution function, for which the relation $M_{n}: M_{w}: M_{z}=$ $(b+1):(b+2):(b+3)$ holds. The molecular weight $\left(12.2 \times 10^{4}\right)$ evaluated from elemental analysis is in accord with this $M_{n}\left(12.3 \times 10^{4}\right)$ value, which supports the conclusion that the molecular weights evaluated from elemental analysis using the number-average molecular weight, $M_{n}=3600$, of polybutadiene can be regarded as the number- average molecular weights.

The thermal helix-coil transition of PBCL and the LBL block copolymers was investigated in a $36: 64$ (v/v) mixture of dichloroacetic acid (DCA) and DCE. Figures 2 and 3 show the temperature dependence of the Moffitt parameter for PBCL and the LBL block copolymers, respectively. It is obvious from these figure that the helix-coil transition temperature $T_{\mathrm{t}}$ shifts toward higher temperature as the molecular weight of PBCL decreases.

Lifson and Roig, ${ }^{10}$ and Nagai ${ }^{11}$ have expressed $T_{\mathrm{t}}$ of a homopolypeptide in powers of the reciprocal number-average molecular weight, $M_{n}{ }^{-1}$,

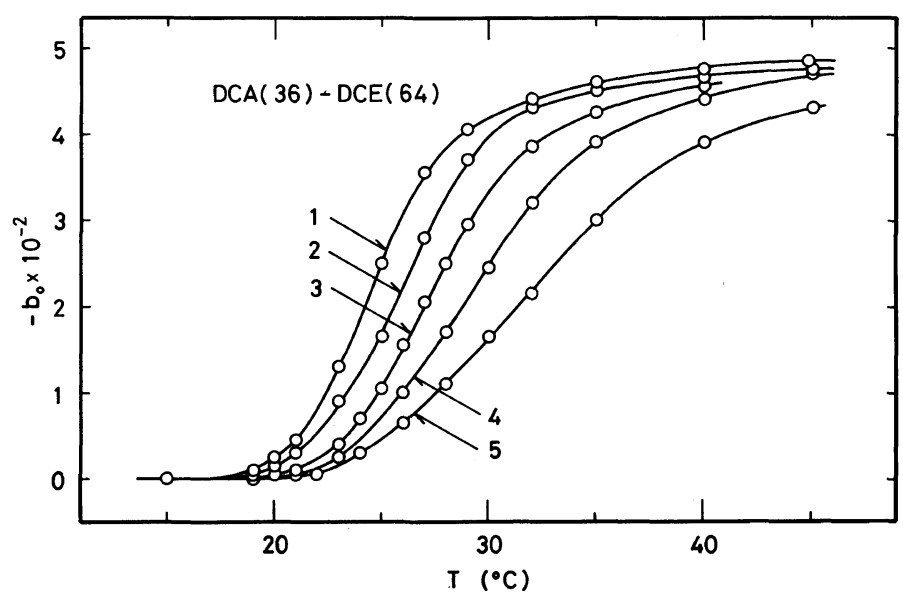

Figure 2. Temperature dependence of the Moffitt parameter $b_{0}$ for PBCL homopolymers of different molecular weights in a 36:64 DCA-DCE mixture. The number-average molecular weights $M_{n}$ are (1), $34.5 \times 10^{4},(2), 20.8 \times 10^{4},(3), 13.9 \times 10^{4},(4), 9.7 \times 10^{4}$, and $(5), 7.0 \times 10^{4}$.

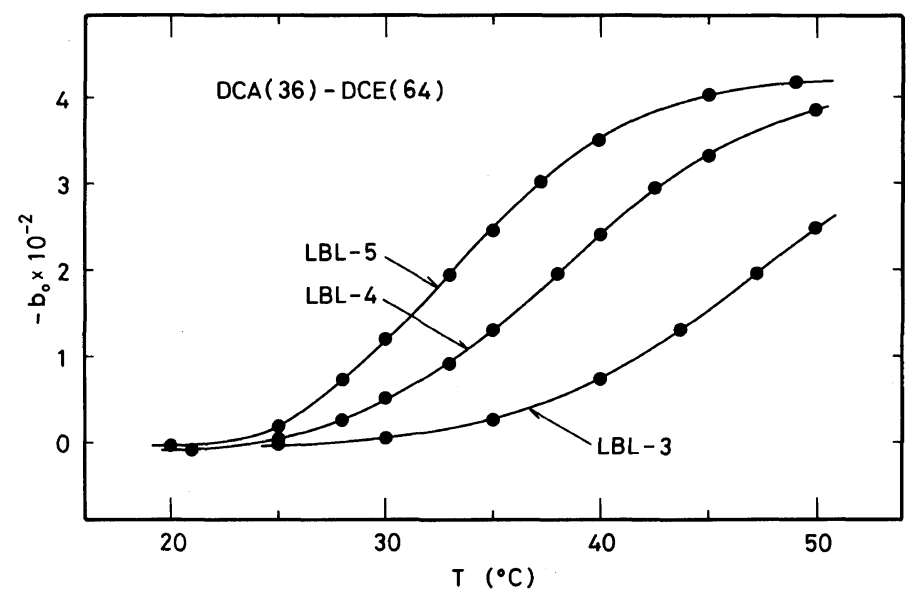

Figure 3. Temperature dependence of the Moffitt parameter $b_{0}$ for the LBL block copolymers in a 36:64 DCA-DCE mixture. 


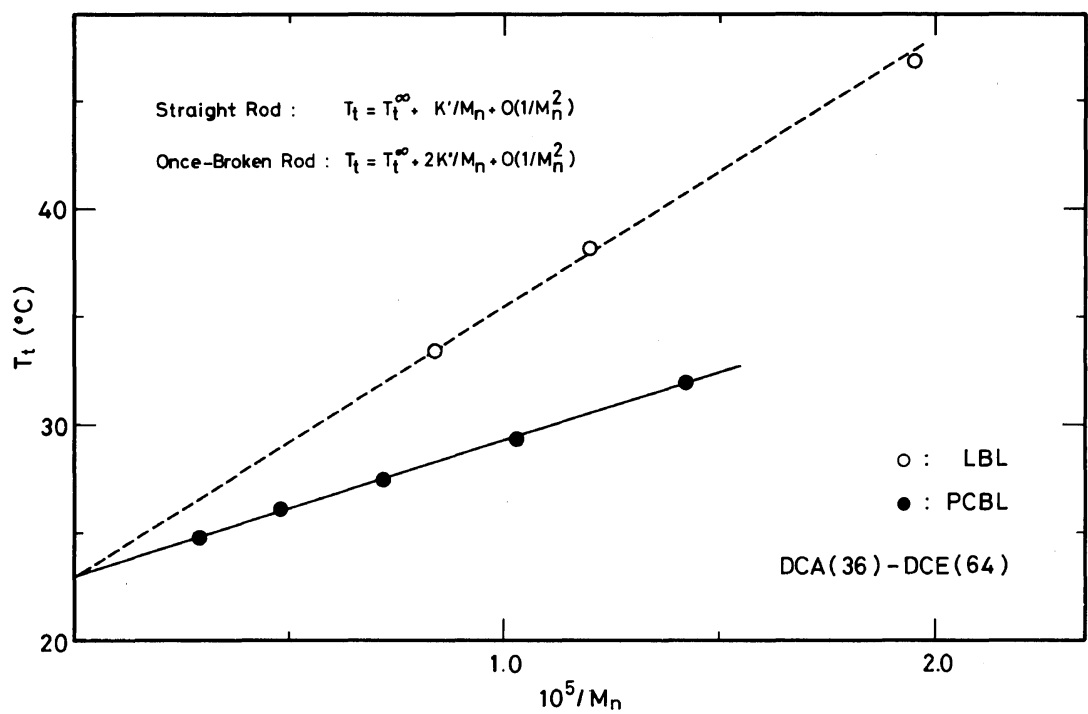

Figure 4. Helix-coil transition temperatures of LBL copolymers (O) and PBCL homopolymers plotted against the reciprocal of the number average molecular weights, $M_{n}{ }^{-1}$.

$$
T_{\mathrm{t}}=T_{\mathrm{t}}^{\infty}+K^{\prime} / M_{n}+0\left(1 / M_{n}^{2}\right)
$$

where $T_{\mathrm{t}}^{\infty}$ is the value of $T_{\mathrm{t}}$ for infinite molecular weight and $K^{\prime}$ is a constant independent of $M_{n}$. Teramoto et al..$^{12}$ have applied the above equation to the case in which a polypeptide molecule has an intrachain defect which will not allow it to participate in the formation of a helix. In such a case, $T_{\mathrm{t}}$ is expressed as,

$$
T_{\mathrm{t}}=T_{\mathrm{t}}^{\infty}+2 \beta K^{\prime} / M_{n}+0\left(1 / M_{n}{ }^{2}\right)
$$

where $\beta$ is a parameter associated with the chemical nature of the defect, and should take a value of unity if the defect acts in the same way as the chain end of the molecule in the formation of the helix.

The LBL block copolymers may be regarded as once-broken rods which may be treated by eq 2 with $\beta=1$. The values of $T_{\mathrm{t}}$ for PBCL and LBL obtained from Figures 2 and 3 are plotted against $M_{n}{ }^{-1}$ in Figure 4. The experimental points for the PBCL homopolymers follow a straight line. The broken straight-line has a slope twice as large as that for the PBCL homopolymers. The experimental points for the LBL block copolymers fall well on this broken straight-line. This suggests that the LBL block copolymers are of the A-B-A tri-block type, consisting of $\alpha$-helical rods (A) of equal length linked to the ends of a random coil chain (B).

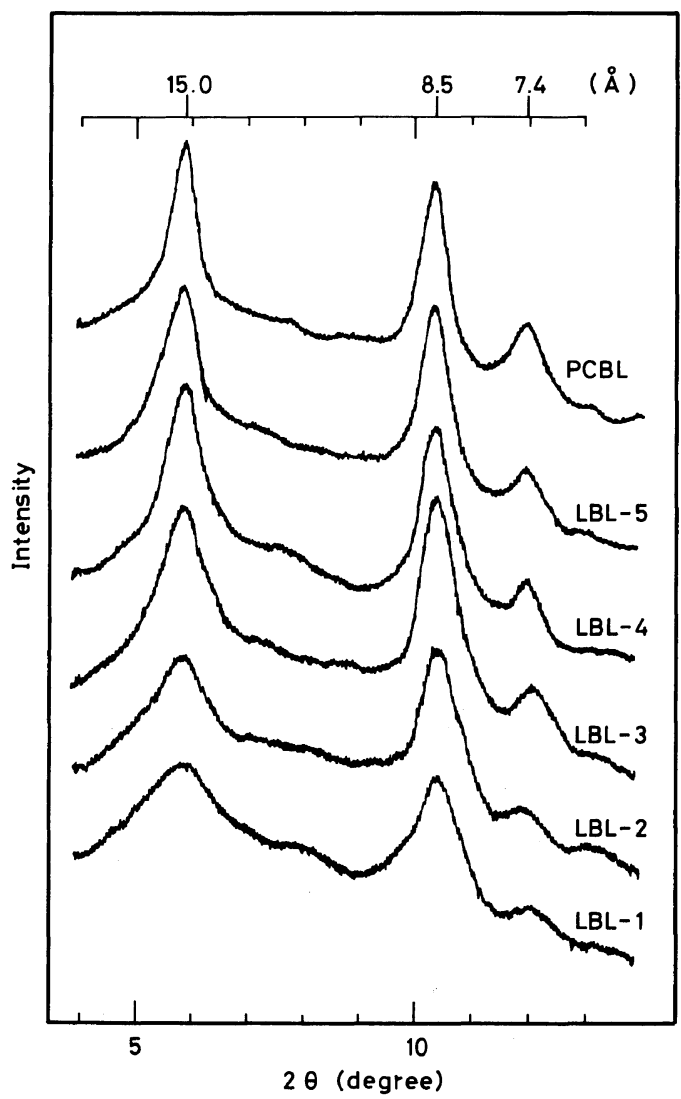

Figure 5. Wide-angle X-ray diffraction profiles of PBCL and LBL block copolymers. 


\section{Structure of Copolypeptides in Solid State}

Figure 5 illustrates the WAXD patterns for the LBL block copolymers and the PBCL homopolymer. The first main reflection of PBCL is very sharp and corresponds to an intermolecular spacing of the $\alpha$-helical chains, which is $15.0 \AA$. Assuming that the $\alpha$-helical rods form the hexagonal packing $(a=b)$ in the films, we could calculate the radius of the $\alpha$-helical rod containing the side-chain and was to be $8.7 \AA$, which is in good agreement with the literature data ${ }^{13}$ obtained from small-angle $\mathrm{X}$-ray scattering for $\operatorname{poly}(\varepsilon-N$-benzyloxycarbonyl-L-lysine) in solution. Regarding the diffraction patterns of the LBL block copolymers, the first main reflections are at the same position as that of $\mathrm{PBCL}$, and become broader as the amount of butadiene increases. Apparently in
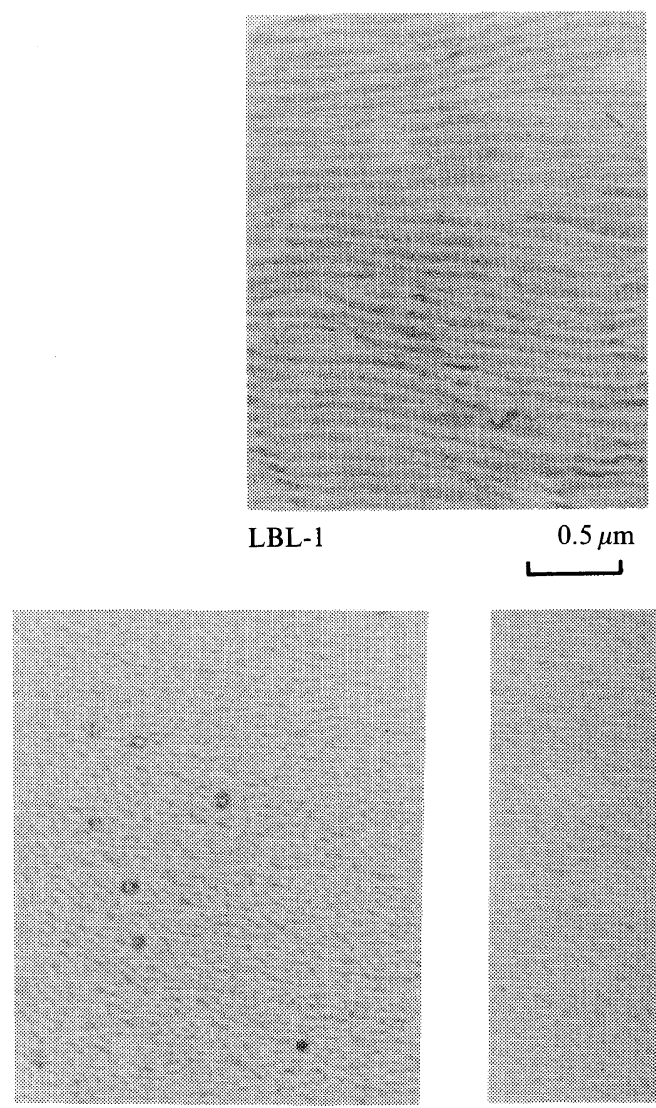

LBL-3

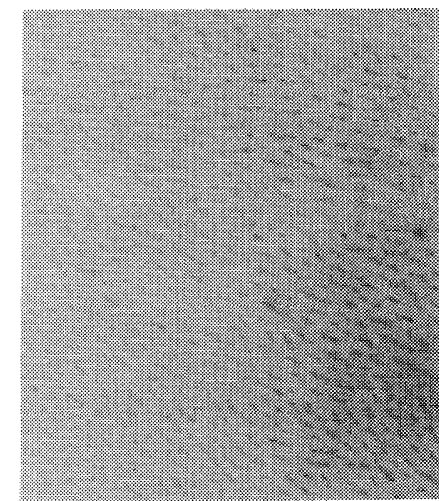

LBL-4 this case, microheterophase separation occurred, and the crystallinity or orientation of the $\alpha$-helical rods diminished.

\section{Morphology}

The morphology of the LBL block copolymers in a membrane was investigated by electron microscopy. The electron micrographs of the LBL membranes show clear microheterophase structures, as illustrated in Figure 6. The dark region in these photographs corresponds to the polybutadiene domain (B domain) stained with osmium tetroxide $\left(\mathrm{OsO}_{4}\right)$ vapor. The B domains of LBL-5, LBL-4 and LBL-3, and LBL-2 and LBL-1 are spherical, cylindrical, and lamella-like micelle, respectively. The shapes and dimensions of the domains are discussed in detail in the succeeding sections.
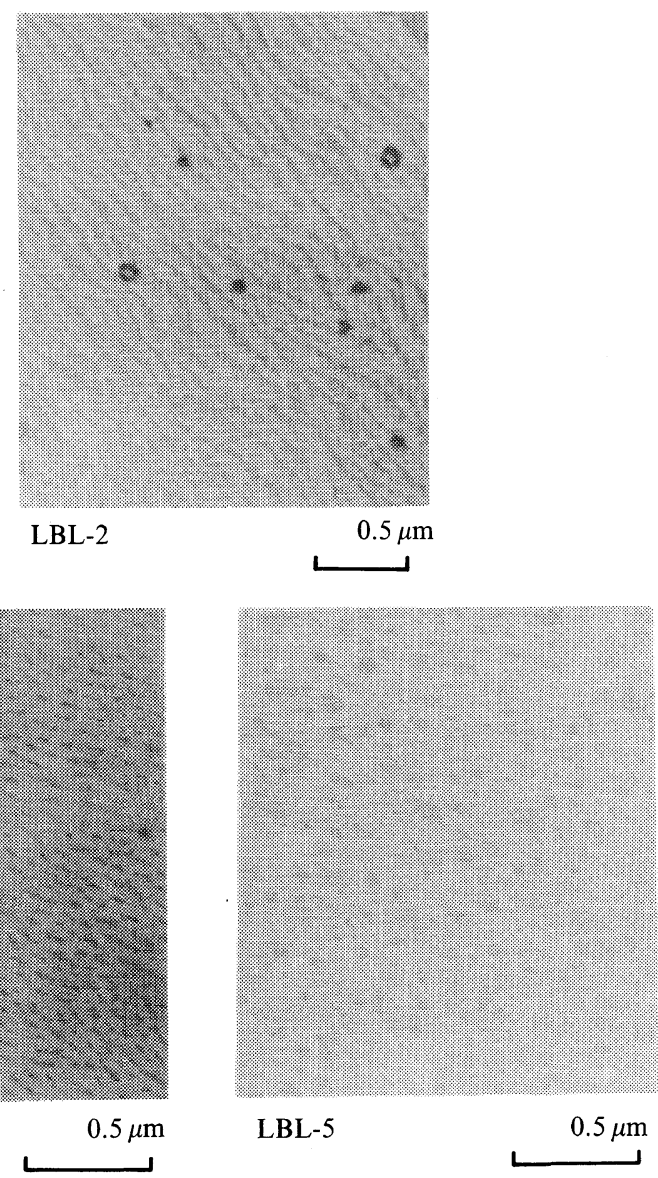

Figure 6. Electron micrographs of the LBL copolymer membranes cast from a $10: 1(\mathrm{v} / \mathrm{v})$ mixture of CF and TFE at $25^{\circ} \mathrm{C}$. The dark portions correspond to PB domains stained by osmium tetroxide. 
Dynamic Mechanical Properties

The dynamic mechanical properties of a twophase system have been successfully described by using Takayanagi's mechanical equivalent model, ${ }^{6,14,15}$ which is the simple model composed of series and parallel elements. Dickie ${ }^{16}$ has shown that Takayanagi's model equation is equivalent to Kerner's equation ${ }^{17}$ assuming that the inclusion phase has the shape of spherical particles and that the Poisson's ratios of the inclusion and matrix phases are equal to 0.5. The dynamic Young's modulus $E_{\mathrm{c}}{ }^{*}$ for Takayanagi's model is given by,

$$
\frac{1}{E_{\mathrm{c}}{ }^{*}}=\frac{\psi}{\lambda E_{2}{ }^{*}+(1-\lambda) E_{1}{ }^{*}}+\frac{1-\psi}{E_{1}{ }^{*}}
$$

where $E_{1}{ }^{*}$ and $E_{2}{ }^{*}$ are the complex moduli of the matrix and the inclusion phase, respectively, $\lambda$ and $\psi$ are the parameters representing the mixing state, and $\lambda \psi$ is equivalent to the volume fraction $\varphi$ of the inclusion phase, i.e., $\varphi=\lambda \psi$. Under the above conditions, $\lambda$ and $\psi$ are expressed as follows ${ }^{14}$.

$$
\begin{aligned}
& \lambda=\frac{2+3 \varphi}{5} \\
& \psi=\frac{5 \varphi}{2+3 \varphi}
\end{aligned}
$$

The $\log E^{\prime} v s$. temperature relations for the LBL-3 block copolymer and the PBCL homopolymer are shown in Figure 7. The dynamic modulus $E^{\prime}$ of polybutadiene in this temperature range was estimated according to the literature value. ${ }^{18}$ In this system, the polybutadiene domain (domain 2) is dispersed in the matrix phase of the PBCL domain (domain 1) as was shown by electron microscopy. The volume fraction $\varphi$ of the polybutadiene component in the LBL-3 block copolymer membrane was estimated to be 0.237 from the results of molecular characterization, assuming that the polybutadiene chains in the solid membrane are in an unperturbed state, i.e., the expansion coefficient $\alpha$ is 1.0. ${ }^{5,19}$ The solid curves in Figure 7, except for those of PBCL and polybutadiene, were calculated by a trial-and-error method. By minimizing the deviation between experimental and calculated curves, $\lambda=0.56$ and $\psi=0.42$ were obtained for the best fitting case (Figure 7). When the shape of the inclusion phase is spherical, we obtain $\lambda=0.54$ from eq 4 using $\varphi=0.237$. The best-fit value, 0.56 , obtained for the LBL-3 block copolymer, is somewhat larger than that for spherical inclusion. This sug-

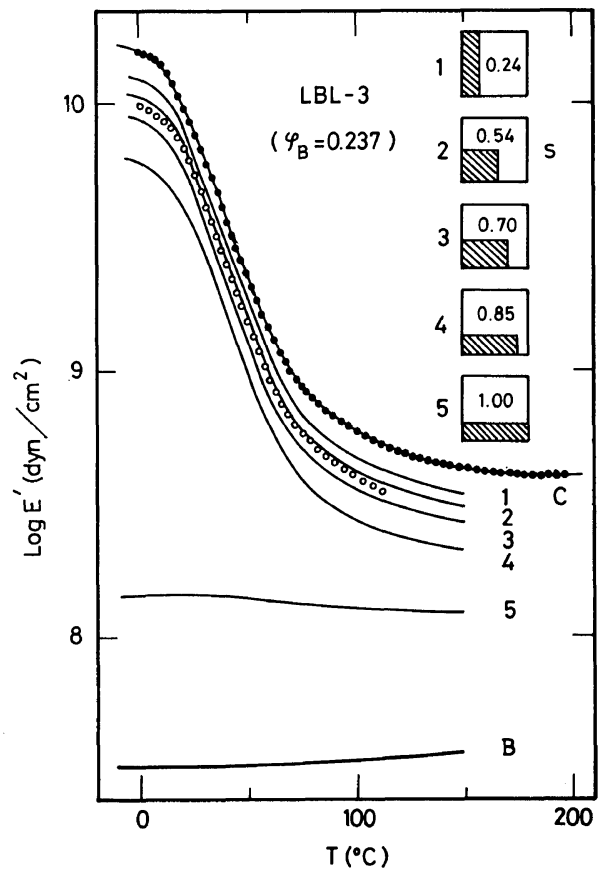

Figure 7. Temperature dependences of $\log E^{\prime}$ for the LBL-3 block copolymer. Calculated curves $1-5$ are compared with experimental data $(O)$. The curve $\mathrm{C}$ denote the experimental results on PBCL homopolymer. The curve $\mathrm{B}$ denotes the literature value for polybutadiene. $^{18}$

gests that the dispersed phase takes on a more extended shape, i.e., the ellipsoidal or cylindrical inclusion phase is expected for the polybutadiene component in the LBL-3 block copolymer membrane; this is in good agreement with observation by electron microscope.

Thermodynamic Investigation for Micelle Dimension

The micelle formation of the A-B-A type block copolymer consisting of an $\alpha$-helical A component and a random coil B component was clarified in the previous paper ${ }^{4}$ by taking into account the conformational parameters of the component block chains. The equations derived therein should be pertinent to this study, since the LBL block copolymers consist of an $\alpha$-helical block and a random coil block. By minimizing the Gibbs free energy $\Delta G$ per unit volume for the micelle formation, we obtained the equilibrium micelle dimensions for each type of micelle models, i.e., sphere $D_{\mathrm{s} \text {, eq }}$, cylinder $D_{\mathrm{c}, \text { eq }}$, and lamella $L_{\mathrm{eq}}{ }^{4}$ : 
Table II. Conformational parameters and dimensions of domains ( $\AA$ )

\begin{tabular}{lcccccl}
\hline \multirow{2}{*}{ Designation } & $\varphi_{\mathrm{B}}$ & $\frac{\mathrm{N}}{\AA^{-3}}$ & $D_{\mathrm{s}, \text { eq }}$ & $D_{\mathrm{c}, \text { eq }}$ & $L_{\text {eq }}$ & $D_{\text {EM }}$ (Micelle) \\
& & & & & 257 & 350 (Lamella) \\
& 0.653 & $3243 \times 10^{-8}$ & & & 301 & 380 (Lamella) \\
LBL-1 & 0.557 & $2764 \times 10^{-8}$ & & 357 & & 400 (Cylinder) \\
LBL-2 & 0.350 & $1738 \times 10^{-8}$ & & 422 & & 460 (Cylinder) \\
LBL-3 & 0.250 & $1243 \times 10^{-8}$ & \multirow{2}{*}{421} & & & 460 (Sphere) \\
LBL-4 & 0.189 & $940 \times 10^{-8}$ & 421 &
\end{tabular}

$$
\begin{aligned}
D_{\mathrm{s}, \text { eq }} & =\left(\frac{8 \Delta W\left\langle r_{\mathrm{B} / 2}^{2}\right\rangle}{k T N}\right)^{1 / 3} \\
D_{\mathrm{c}, \mathrm{eq}} & =\left(\frac{16 \Delta W\left\langle r_{\mathrm{B} / 2}^{2}\right\rangle}{3 \varphi_{\mathrm{B}}^{1 / 2} k T N}\right)^{1 / 3} \\
L_{\mathrm{eq}} & =\left(\frac{8 \Delta W\left\langle r_{\mathrm{B} / 2}^{2}\right\rangle}{3 \varphi_{\mathrm{B}}^{2} k T N}\right)^{1 / 3}
\end{aligned}
$$

$\Delta W$ is the interfacial free energy per unit area of $\mathrm{A} / \mathrm{B}$ interface, $\left\langle r_{\mathbf{B} / 2}^{2}\right\rangle$ is the mean-square end-to-end distance of the $\mathrm{B} / 2$ chain, $k$ is the Boltzmann constat, $T$ is the absolute temperature, $N$ is the number of junction points between $A$ and $B$ block per unit volume of micelle, i.e., twice the number of block copolymers per unit volume of micelle, and $\varphi_{\mathrm{B}}$ is the volume fraction of the domain occupied by B blocks relative to the total volume occupied by the copolymer. In this study, we obtained $\Delta W=$ $21.1 \times 10^{-16} \operatorname{erg} \AA^{-2}$ for the PBCL-PB interface. ${ }^{20}$ Table II summarizes the data for the microheterophase structure of the LBL block copolymers. All calculations were made using eq 6, 7, and 8 in the same manner as described in our previous paper. ${ }^{4} D_{\mathrm{EM}}$ is the observed micelle dimensions, and the shape of micelles as determined by electron microscope is listed in the last column of Table II. It is seen that the shape and size of the micelles calculated thermodynamically agree with the electron microscope observation.

In summary, we synthesized A-B-A type block copolymers consisting of poly $(\varepsilon-N$-benzyloxycarbonyl-L-lysine) as the A component and polybutadiene as the $\mathrm{B}$ component, with the hope of acquiring more information on the A-B-A type block copolymers of $\alpha$-amino acid and butadiene components. We characterized the samples and established their microheterophase structure by dynamic mechanical measurements and thermodynamic considerations.
Acknowledgments. We wish to thank Drs. K. Rieu and R. Drake, the B. F. Goodrich Chem. Co., for kindly supplying the amine-terminated polybutadiene. We also extend our appreciation to Mr. S. Yamaguchi, Central Research Laboratory, Daikin Kogyo Co., for helping with the electron microscopy, and to Associate Professor K. Nakamae, Department of Industrial Chemistry, Kobe University, for his assisting with wide angle $\mathrm{X}$-ray diffraction.

\section{REFERENCES}

1. B. Gallot et al., Makromol. Chem., 177, 1889 (1976); 177, 2569 (1976); 178, 1595 (1977); 178, 1641 (1977).

2. A. Douy and B. Gallot, Polym. Eng. Sci., 17, 523 (1977).

3. A. Nakajima, T. Hayashi, K. Kugo, and K. Shinoda, Macromolecules, 12, 840 (1979).

4. A. Nakajima, K. Kugo, and T. Hayashi, Macromolecules, 12, 844 (1979).

5. A. Nakajima, K. Kugo, and T. Hayashi, Polym. J., 11, 995 (1979).

6. M. Takayanagi, Mem. Fac. Eng., Kyushu Univ., 23, 41 (1963).

7. E. R. Blout and R. H. Karlson, J. Am. Chem. Soc., 78, 941 (1956).

8. A. Cosani, E. Peggion, E. Scoffone, and A. Portolan, Biopolymers, 4, 695 (1966).

9. T. Hayashi, Ph.D. thesis, Kyoto University, 1973, p 84.

10. S. Lifson and A. Roig, J. Chem. Phys., 34, 1963 (1961).

11. K. Nagai, J. Chem. Phys., 34, 887 (1961).

12. A. Teramoto, T. Yamashita, and H. Fujita, J. Chem. Phys., 46, 1919 (1967).

13. Y. Ishimuro, F. Hamada, and A. Nakajima, Macromolecules, 11, 382 (1978).

14. M. Takayanagi, S. Uemura, and S. Minami, $J$. Polym. Sci., C, 5, 113 (1964).

15. S. Uemura and M. Takayanagi, J. Appl. Polym. Sci., 


\section{Microphase Structure of Block Copolymers}

10, 113 (1966).

16. R. A. Dickie, J. Appl. Polym. Sci., 17, 45 (1973).

17. D. H. Kerner, Proc. Phys. Soc., B, 69, 808 (1956).

18. H. Keskkula and S. G. Turley, J. Polym. Sci. B, 7, 697 (1969).

19. H. Hayashi, F. Hamada, and A. Nakajima, Macro- molecules, 7, 959 (1974).

20. A. Nakajima, K. Kugo, T. Hayashi, and H. Sato, "Biomedical Polymers: Polymeric Materials and Pharmaceuticals for Biomedical Use," E. P. Goldberg and A. Nakajima, Ed., Academic Press Inc., 1980, p 243. 